\title{
Hypoglossal Nerve Palsy as a Complication of an Anterior Approach for Cervical Spine Surgery
}

\author{
Tatsuya Yasuda, Daisuke Togawa, Tomohiko Hasegawa, Yu Yamato, \\ Sho Kobayashi, Hideyuki Arima, Yukihiro Matsuyama \\ Department of Orthopaedic Surgery, Hamamatsu University School of Medicine, Hamamatsu, Japan
}

A recurrent laryngeal nerve injury is known as a complication referring to an anterior cervical spine surgery. However, hypoglossal nerve injury is not well known yet. Herein we report a rare case of a 39-years-old male with a hypoglossal nerve injury after C3/4 osteophyte resection with Smith-Robinson approach. In this case there appeared difficulties of articulation and tongue movement with deviation of the tongue to the left side after the surgery and we diagnosed a hypoglossal nerve injury due to retraction against the nerve during the operation. During the operative approach to the upper cervical spine we had to retract the internal carotid artery and the soft tissue to reach the vertebrae. This retract was the cause of the hypoglossal nerve injury. A gently traction and intermittent release is important to avoid a hypoglossal nerve damage.

Keywords: Hypoglossal nerves; Cervical vertebrae

\section{Introduction}

The Smith-Robinson approach is a popular approach for an anterior cervical spine surgery [1]. A dysphagia is a well-known complication after anterior cervical spine surgery and a recurrent laryngeal nerve injury is known as origin of this complication. However, a hypoglossal nerve injury is only known as a complication after soft tissue surgery in the upper anterior aspect of the neck by anterior retropharyngeal approach, but not as a complication of a cervical spine surgery. We report a rare case of hypoglossal nerve palsy after anterior cervical spine surgery.

\section{Case Report}

The patient was a 39-years-old man who had difficul- ties to swallow solid food and liquid for 2 years. At the same time he was diagnosed with sleep apnea syndrome. Chronic sinusitis was told as a factor of dysphagia and sleep apnea syndrome. An osteophyte of the anterior cervical spine was additionally diagnosed by radiograph. Thereafter, the patient visited our hospital. It was found that the dysphagia was increased by flexion and extension of the neck. Particularly, the patient aspirated fluid by extension of the neck. He often awoke by aspiration of saliva when he was sleeping. The patient showed no neurological deficit. Radiograph and computed tomography revealed an osteophyte on $\mathrm{C} 3 / 4$ on the anterior vertebrae (Figs. 1, 2). There was no osteophyte at another level. By videofluorography, barium stayed at the level of the osteophyte (Fig. 3) and entered into the trachea by neck extension. A dysphagia caused by an osteophyte of C3/4 anterior vertebrae was diagnosed. An osteophyte

Received Feb 6, 2014; Revised Mar 17, 2014; Accepted Apr 11, 2014

Corresponding author: Tatsuya Yasuda

Department of Orthopaedic Surgery, Hamamatsu University of Medicine,

1-20-1 Handayama, Higashi-ku, Hamamatsu 431-3192, Japan

Tel: +8153-435-2299, FAX: +8153-435-2296, E-mail: t.yasuda@hama-med.ac.jp 


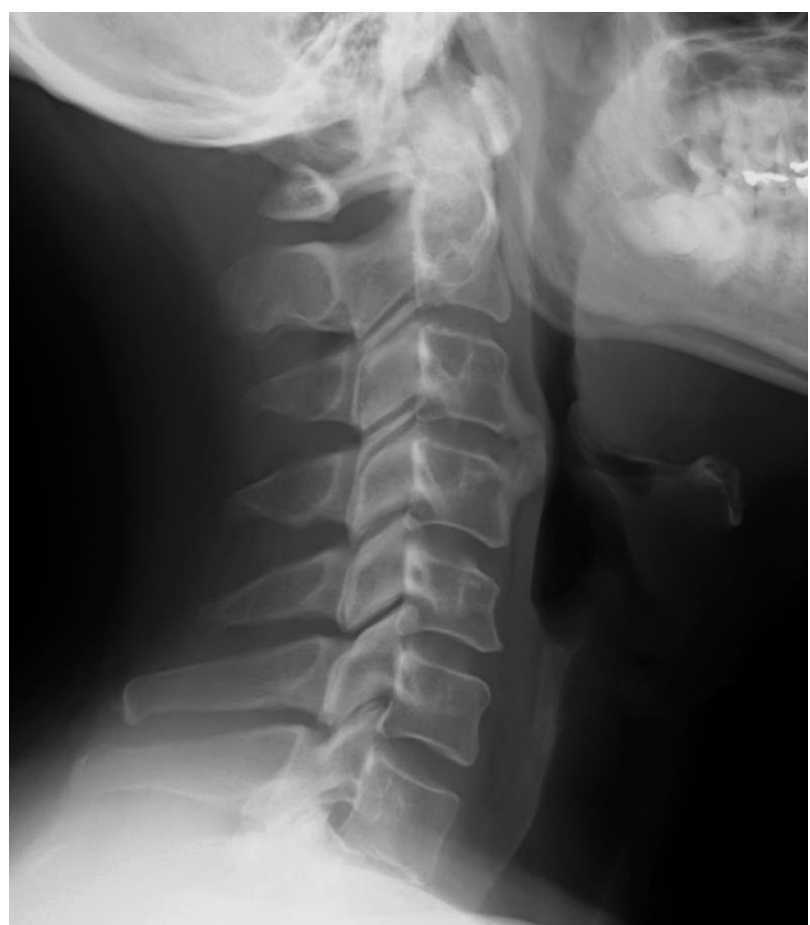

Fig. 1. Lateral radiography of the cervical spine showing an anterior osteophyte at C3/4.

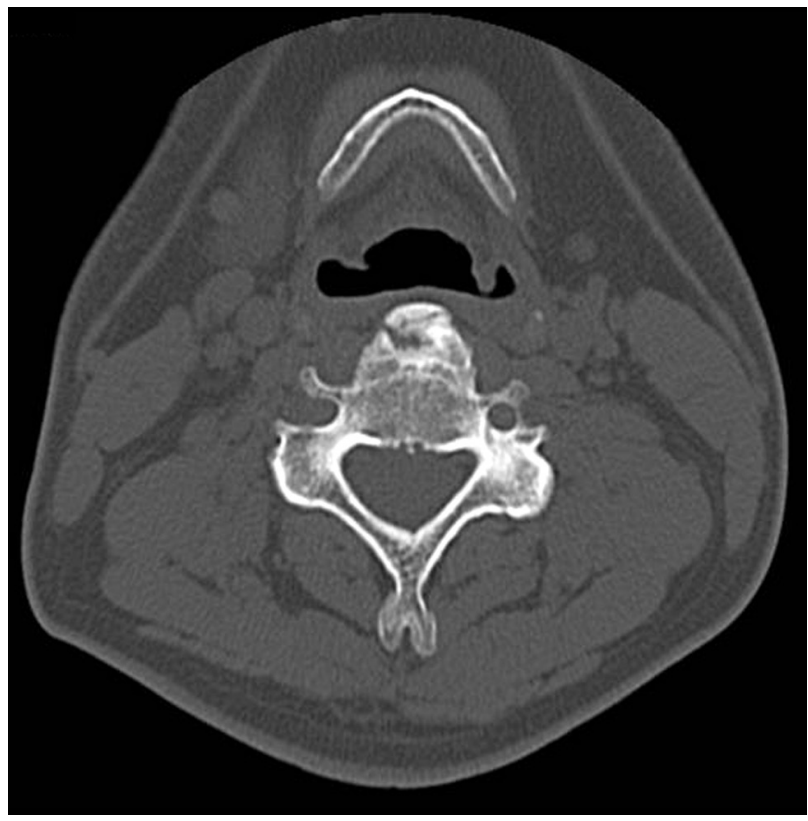

Fig. 2. A computed tomography scan showing the C3/4 osteophyte pushing the pharyngeal wall.

resection was done through a left anterior oblique incision in the prone position. Esophagus and muscle were retracted by surgical assistants without self retaining retractor. The osteophyte on C3/4 was removed using ron-

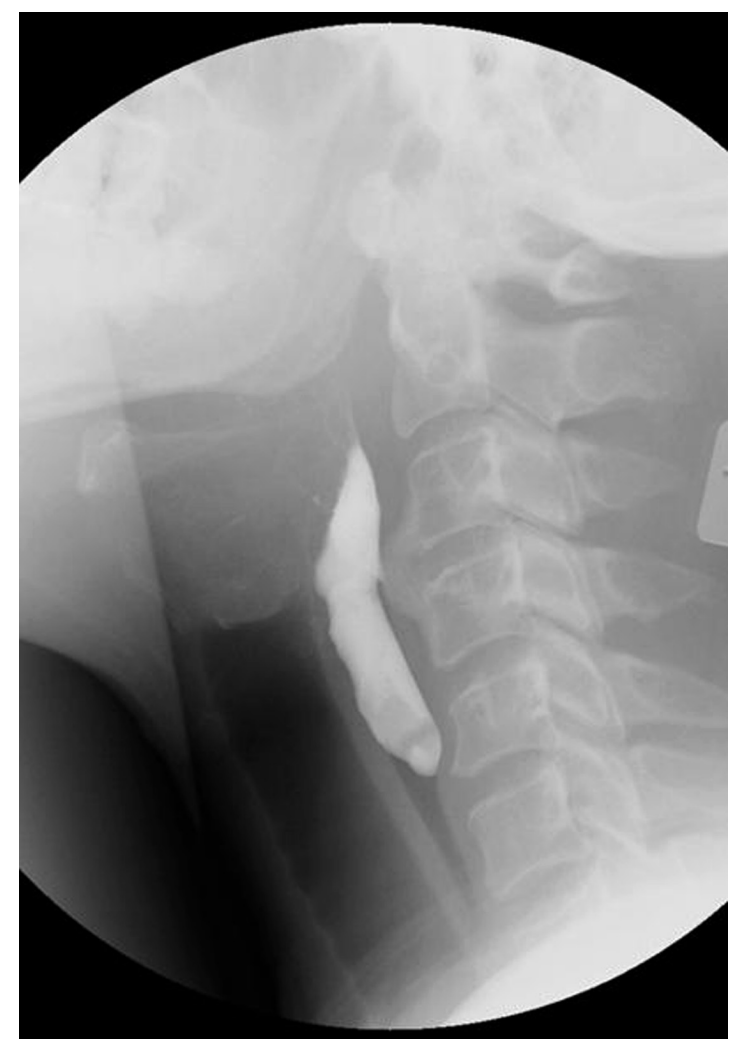

Fig. 3. Preoperative videofluorographic examination of barium swallowing.

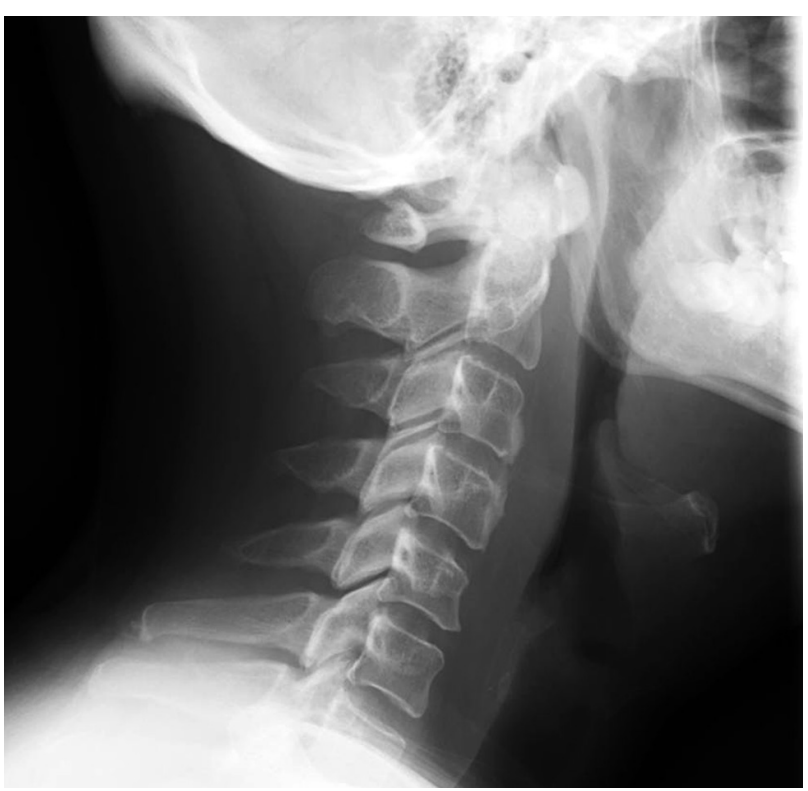

Fig. 4. Postoperative lateral radiography of the cervical spine.

geurs and surgical burr (Fig. 4). The patient showed still a dysfunction of swallowing postoperatively. Additionally, he had difficulties in the articulation and tongue movement with a deviation of the tongue to the left 

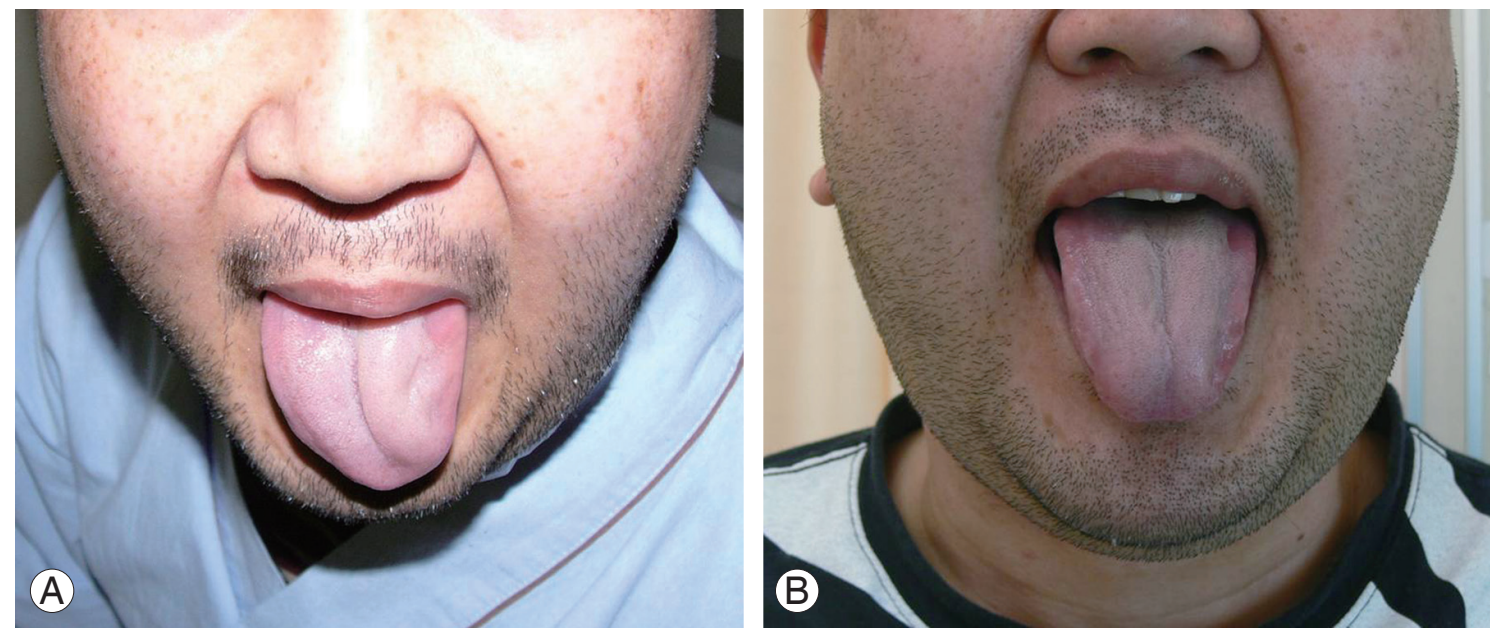

Fig. 5. (A) Tongue deviation to the left side caused by hypoglossal nerve palsy one day after surgery. (B) Hypoglossal nerve palsy had recovered one year after surgery.

side (Fig. 5A). This was thought to be hypoglossal nerve palsy due to an excessive retraction against the nerve during the anterior cervical surgery. About three weeks after surgery, the symptoms gradually improved without any specific treatment. The hypoglossal nerve palsy and dysphagia were completely recovered at the postoperative observation 1 year later (Fig. 5B). The patient and his family were informed and gave their consent for the submission of the data for publication.

\section{Discussion}

Graham [2] reported an overall incidence of $1.04 \%$ for neurologic complications in 5,356 cases of cervical spine surgery. Particularly, a recurrent laryngeal nerve injury is common as a complication after anterior cervical spine surgery. However, there is only one report known of hypoglossal nerve palsy after anterior cervical spine surgery through Smith-Robinson approach [3]. The case reported a complication after upper cervical spine surgery.

The patient with hypoglossal nerve injury presented with a dysphagia. However, a dysphagia also often occurs as complication after an anterior cervical spine surgery [4-6]. Swallowing is divided into three phases; oral, pharyngeal, and esophageal. A dysphagia is a popular failure in the pharyngeal phase which occurs due to recurrent laryngeal nerve injury or hematoma after an anterior cervical spine surgery. The hypoglossal nerve plays an important role in the oral phase. Therefore, deficits in the oral phase may suggest damage to the hypoglossal nerve.
If there is hypoglossal nerve palsy, the tongue will point toward the palsy side. This symptom is the key to the diagnosis of a hypoglossal nerve injury.

The hypoglossal nerve is the twelfth cranial nerve. Anatomically, the hypoglossal nerve arises from the hypoglossal nucleus and passes through the hypoglossal canal and between the internal carotid artery and internal jugular vein. Usually, the hypoglossal nerve does not appear in the operative field during anterior cervical spine approach. But in surgical approaches to the upper cervical spine, to reach the vertebrae it is necessary to retract internal carotid artery to the outside and soft tissue to the inside. This retraction could be the cause of hypoglossal nerve injury in our presented case. Additionally, the hypoglossal nerve may transverse the path within $5 \mathrm{~mm}$ of the $\mathrm{C} 2 / 3$ level [4]. Therefore, it is necessary to be careful during the retraction to the cranial side. A gentle retraction and an intermittent release are important to avoid hypoglossal nerve damage.

\section{Conflict of Interest}

No potential conflict of interest relevant to this article was reported.

\section{References}

1. Smith GW, Robinson RA. The treatment of certain cervical-spine disorders by anterior removal of the intervertebral disc and interbody fusion. J Bone Joint 
Surg Am 1958;40:607-24.

2. Graham JJ. Complications of cervical spine surgery. A five-year report on a survey of the membership of the Cervical Spine Research Society by the Morbidity and Mortality Committee. Spine (Phila Pa 1976) 1989;14:1046-50.

3. Sengupta DK, Grevitt MP, Mehdian SM. Hypoglossal nerve injury as a complication of anterior surgery to the upper cervical spine. Eur Spine J 1999;8:78-80.
4. Haller JM, Iwanik M, Shen FH. Clinically relevant anatomy of high anterior cervical approach. Spine (Phila Pa 1976) 2011;36:2116-21.

5. Martin RE, Neary MA, Diamant NE. Dysphagia following anterior cervical spine surgery. Dysphagia 1997;12:2-8.

6. Park SH, Sung JK, Lee SH, Park J, Hwang JH, Hwang SK. High anterior cervical approach to the upper cervical spine. Surg Neurol 2007;68:519-24. 